\title{
Selective serotonin reuptake inhibitor-induced cell death: in search of a mechanism
}

\author{
Christina Glöckel', Christoph Minichsdorfer', Gerhard F Ecker², Michael Freissmuth ${ }^{1 *}$ \\ From 16th Scientific Symposium of the Austrian Pharmacological Society (APHAR) \\ Vienna, Austria. 25-27 November 2010
}

\section{Background}

Selective serotonin reuptake inhibitors (SSRIs) have been observed to drive programmed cell death in Burkitt lymphoma cells. Accordingly, they were proposed as a new modality in the treatment of Burkitt lymphoma. However, they induce apoptosis with little if any appreciable selectivity. Actually, the selectivity appears to be so low that SSRIs can also kill protozoa (such as Trichomonas vaginalis). Acetylated versions of SSRIs, which do not inhibit serotonin reuptake, kill cells in concentration ranges comparable to those of their non-acetylated original versions. Thus, the obvious SSRI target, SERT, is not required for cell killing.

\section{Methods}

For cell viability assays, HEK293 cells and T. vaginalis were incubated with SSRIs or related compounds and subsequently analyzed for propidium iodide-positive cells using flow cytometry. Caspase activation was measured in cell lysates as fluorescence intensity using cleavable fluorogenic substrates of caspases $3 / 7,4,8$ and 9 , respectively. Activation of autophagy and endocytosis was evaluated by immunocytochemistry and by expression of fluorescent labelled markers in HEK293 cells visualized by confocal microscopy.

\section{Results and conclusions}

By testing various SSRIs and tricyclic antidepressants, we found a significant correlation in their ability to kill HEK293 cells and T. vaginalis. In search of a mechanism of SSRI-induced cell killing, we found that (i) SSRIs cause activation of caspases $3 / 7,4,8$ and 9 . This is blocked by z-vad-fmk (caspase inhibitor used as internal control) and by the vacuolar ATPase inhibitor bafilomycin A1, but not by salubrinal, eeyarestatin I and bongkrekic acid; (ii) paroxetine and compounds identified by a chemical library screen (REM14 and REM25) lead to accumulation of vacuoles within cells; paroxetineinduced vacuole formation is inhibited by bafilomycin A1; (iii) paroxetine-induced vacuoles stain for Rab4 and Rab7 (markers for early and late endosomes), but not for LC3B (a marker for autophagosomes). The structure-activity profile for SSRIs and related compounds will be verified by extracting the pharmacophore and by chemical library screens. The additional goal is to identify compounds that block the action of SSRIs and to thereby define their site of actions.

\section{Acknowledgements}

This work is supported by PLACEBO of the GEN-AU programme of the Austrian Federal Ministry of Science and Research.

\section{Author details}

'Institute of Pharmacology, Center of Physiology and Pharmacology, Medical University of Vienna, 1090 Vienna, Austria. ${ }^{2}$ Department of Medicinal Chemistry, University of Vienna, 1090 Vienna, Austria.

Published: 16 November 2010

doi:10.1186/1471-2210-10-S1-A28

Cite this article as: Glöckel et al:: Selective serotonin reuptake inhibitorinduced cell death: in search of a mechanism. BMC Pharmacology 2010 10(Suppl 1):A28.

\footnotetext{
* Correspondence: michael.freissmuth@meduniwien.ac.at

${ }^{1}$ Institute of Pharmacology, Center of Physiology and Pharmacology, Medical University of Vienna, 1090 Vienna, Austria

Full list of author information is available at the end of the article
} 